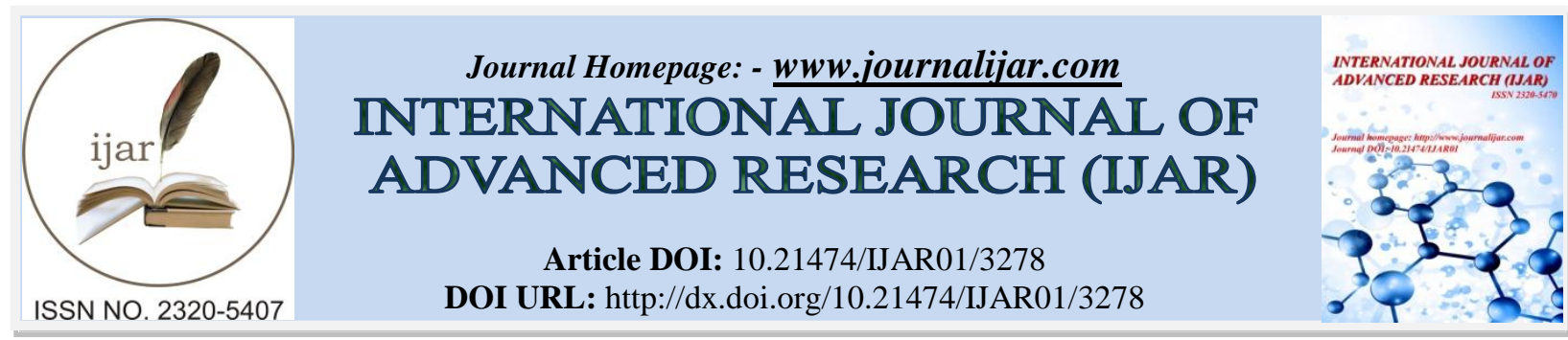

RESEARCH ARTICLE

\title{
USERS EXPERIENCES AND PERCEPTIONS ON MATERNAL HEALTHCARE SERVICES IN SIAYA COUNTY, WESTERN KENYA: A QUALITATIVE STUDY.
}

Obinge Elizabeth Omondi ${ }^{1,3}$, Fred A. Amimo ${ }^{1}$, Omondi S. Owino ${ }^{3}$ and Asito Stephen Amolo ${ }^{2}$.

1. Department of Public Health, School of Health Sciences, Jaramogi Oginga Odinga University of Science and Technology.

2. Department of Biological Sciences, School of Biological and Physical Sciences, Jaramogi Oginga Odinga University of Science and Technology.

3. Department of Health, County Government of Siaya.

\section{Manuscript Info}

Manuscript History

Received:12 December 2016

Final Accepted: 27 January 2017

Published: February 2017

Key words:-

Skilled maternal healthcare services, community maternal healthcare, continuum of care, utilization, suggestions.

\section{Abstract}

Background: Proper utilization of maternal healthcare services greatly reduces maternal and newborn morbidity and mortality. However, utilization of such care is poor in Siaya County western Kenya. This study explored the user's experiences and perception of maternal healthcare services, and gathered suggestions for optimizing utilization of maternal healthcare services in Siaya County.

Methods: We conducted a cross sectional qualitative study from January to March 2015 in 8 selected community health units. Eight focus group discussions and 14 in depth interviews were carried out involving a total of 98 respondents.

Results: Our results determined that the major barriers affecting utilization of maternal healthcare services included inadequate awareness of availability of the services and their importance, limited availability of skilled maternal healthcare services, negative provider's attitude, maternal fears, weak facility-community linkage and no evidence of maternal healthcare continuum.

Conclusion: To bridge these gaps there is need to intensify awareness creation on available of skilled maternal healthcare services and their importance, avail 24 hours skilled delivery services, provide incentives to mothers and promotion of positive attitude amongst skilled providers. Important is also empowerment of community health volunteers (CHVs) to be able to identify pregnant mothers early through pregnancy testing at community and enhancing maternal healthcare continuum by providing evidence based community maternal health services.

Copy Right, IJAR, 2017,. All rights reserved.

\section{Introduction:-}

Utilization of skilled maternal healthcare services (during pregnancy, childbirth and post partum period) are amongst the major interventions aimed at reducing maternal and newborn mortality worldwide [1, 2]. Although since 1990, the world has seen a $44 \%$ decline in the maternal death ratio, still, 800 women die from preventable causes related to pregnancy and childbirth every day, with most of the deaths occurring among women of reproductive age in the 
developing countries of the world [3, 4]. Previous studies have shown that a woman's lifetime risk of dying in pregnancy or childbirth in developed countries is 1 in 3700, the risk of dying in sub Saharan Africa is 1 in 38 which is about 1 (one) woman every two minutes[3] and for every woman who dies 20 or 30 encounter complications with serious or long-lasting consequences. These deaths and injuries are partly due to inadequate utilization and poorquality maternal health care, -antenatal care, -skilled attendance at birth and postnatal care [5, 6] and are entirely preventable through increased utilization of skilled maternal healthcare services [3]. In addition these mortality have been associated with maternal education, maternal age and autonomy of the mother [7-9]. Several studies have shown that skilled birth attendants during labour, delivery and the early postpartum period can reduce significant number of maternal and newborn deaths through provision of timely obstetric and newborn care [10]. Owing to the central role of professional care at birth, skilled attendance was chosen as an indicator for monitoring progress towards maternal health MDG-5 of reducing maternal mortality ratio by three quarters between 1990 and 2015 [7].

Kenya was among the top ten countries that contributed to $58 \%$ of the global maternal deaths reported in 2013 [3]. Maternal mortality levels in Kenya have remained high (495 per 100,000 live births) [11] with slightly over 6,000 women dying every year due to pregnancy related conditions despite the launch of Safe Motherhood campaigns twenty years ago [12]. According to KDHS, 2014; the proportion of women attending antenatal clinic at least once in Kenya are $95 \%$, four times as per WHO recommendation are 58\% and postnatal care within two days of delivery at $51 \%$. Moreover, unskilled persons attend to about $50 \%$ of deliveries by Kenyan women [12]. The low utilization of skilled maternal healthcare services has been attributed to cost of service, distance, quality of service, culture, birth order, living conditions of the mother, type of place or residence, level of education, wealth and weak healthcare infrastructure [10, 12-14]. Siaya County is one of the counties in Kenya with high maternal mortality ration (MMR) that is above the national average of 495 deaths for every 100,000 live births, with most of these deaths being attributed to home deliveries and low utilization of skilled maternal healthcare services [11]. The high maternal mortality ratio in Siaya County has been mainly attributed to low use of maternal healthcare services [15]. Data from District Health Information Software (DHIS) 2014 indicate that the proportion of women attending at least one ANC visit are 85\%, those attending four ANC visits are 45\%, 52\% receive skilled care during delivery while only $30 \%$ receive postnatal care within two days after delivery [11]. Consensus is now that the continuum of care for reproductive maternal and child health $(\mathrm{RMNCH})$ includes the seamless and integrated service delivery for women and children throughout the life cycle-from pregnancy to delivery and the immediate postnatal and childhood period, and across all places of care [16]. In an effort to enhance maternal healthcare continuum and create demand for utilization of skilled healthcare services, Siaya County scaled up community health services in the whole County. However, despite massive investments in the strategy, some health indicators including maternal and child health have not yielded positive results due to low uptake of maternal healthcare services.

Although previous studies done in Siaya County associated low uptake of maternal healthcare with the following factors; Poor communication between health workers and women, cultural and religious beliefs, uncertainty about pregnancy, distance to health facilities and quality of ANC services among others [17, 18]. The 100\% scale up of community health workers and services in Siaya County ought to have addressed most of issues mentioned above through regular interaction between $\mathrm{CHW}$ s and pregnant women at the household level and regular engagement with skilled health providers during review and dialogue meetings. However DHIS, 2014 shows that utilization of skilled maternal healthcare services in the county is still low, suggesting that there may be context-specific factors leading to low uptake of these services. Together these data indicate that there is need to identify barriers, understand the perception of service users and providers regarding those barriers and their suggestions for optimizing utilization of skilled maternal healthcare services, and to develop strategies to improve women's access to and utilization of delivery care services. Therefore, the present study aimed to explore how service users (mothers) and providers (health workers and members of health facility management committee) perceive barriers to skilled birth care and their suggestions on strategies for optimizing utilization skilled maternal healthcare services.

\section{Methods:-}

\section{Study site:-}

Siaya County is amongst the top ten Counties with the largest number of maternal deaths (691/100,000 live births) in Kenya which surpass the country maternal mortality rate (488/100,000 live births) [19, 20].These deaths are due to causes directly related to pregnancy and childbirth. Although Siaya County has made efforts to implement many policies and strategies to increase utilization of skilled maternal healthcare services and reduce maternal deaths from preventable conditions the progress has remained slow[15].The slow progress has been attributed to poor communication between health workers and women, cultural and religious beliefs, uncertainty about pregnancy, 
distance to health facilities, quality of ANC services with low uptake of maternal health care services, and breaks in the continuity of care from preconception through antenatal, intra-partum and postnatal periods [21-23]. The County has 6 sub-counties, 179 sub-locations, 198 Community Units and 30 administrative wards. It has a total population of $\sim 955,000$ with inhabitants being predominantly of Luo ethnic extraction.

\section{Sampling strategy:-}

This study used a multi-stage simple random sampling method to select respondents. First 8 health facilities were selected from a sampling frame of all the health facilities (128) in the six sub-counties. Names of the health facilities per Sub County were drafted on pieces of folded paper and put in six boxes and thoroughly churned with each box having the names of all government health facilities per Sub County. One health facility was then randomly picked from each box. The health facility picked included Chianda Dispensary in Rarieda Sub County, Ligala Dispensary in Ugenya Sub County, Bondo Hospital in Bondo Sub County, Sikalame Health Center in Ugunja Sub County, Umalla Dispensary in Alego Sub County and Akalla Health center in Gem Sub County. Secondly, having had a target of 50 pregnant mothers per link health facility, it meant that for the study to achieve the recommended target of 400 , an additional of 2 more health facilities had to be included. We thus placed all the remaining health facilities from the six boxes were placed in one box and thoroughly churned then two health facilities were randomly selected to add to the already selected six to make eight (8). The health facilities picked this time were Midhine and Gongo Dispensary all from Gem Sub County. The third stage involved identifying the community units linked to the selected health facilities. Those identified included; West Katweng'a Community Unit (CU) linked to Chianda Dispensary, Gongo CU linked to Gongo Dispensary, Ligala CU linked to Ligala Dispensary, Nyawita CU linked to Bondo Hospital, Umalla CU linked to Umalla Dispensary, Ndori CU linked to Akalla Health center, Midhine CU linked to Midhine Dispensary and Yiro West CU linked to Sikalame Health Center. It happened that Umalla Dispensary had two CUs linked to the facility (Umalla and Ulafu CUs) and thus Ulafu CU was also included in the study. Stage four involved identification of all the Community Health Volunteers (CHWs) from the selected Community Units to be key informant respondents to the study. Each CU has an average of 10 Community Health Volunteers (CHWs) and all accepted to participate in the study.

\section{Study population:-}

Study participants comprised service users and service providers. Specifically, a representative sample of 9 to 12 mothers within the reproductive age group in every selected community unit participated in the focus group discussions. Others included in the study as key informants were 14 skilled health providers working at the link health facilities, the health managers and the leaders in the community.

\section{Data collection:-}

We recruited eight field researchers to conduct FGDs in the community units (CUs). We developed separate guidelines for FGDs with health service providers and users, and all field researchers attended a two-day training session. To explore the social context, cultural issues, and concerns related to SBA utilization, we conducted eight focus group discussions (FGDs) and 14 in depth interviews involving a total of 98 respondents between from January to March 2015 in 8 selected community health units. During data collection, a focus group discussion guide and semi-structured key informant interview guide were used. Face to face in depth interviews were also conducted both at the health facilities and the communities. Each focus group consisted of 9 to 12 people which represent the ideal size of a focus group. The focus group discussions (FGD) were run by a facilitator with the responsibility to apply the appropriate working group techniques. The facilitator was required to provide equal opportunities for communication to all subgroups of patients and professionals. There were 8 target focus groups in total: one in each of the six sub counties in Siaya County. The women were asked to describe their perception of skilled maternal healthcare services. The women were asked about maternal healthcare services offered at the link health facility and the community. They were also asked about maternal healthcare continuum and utilization situation; suggestions for enhancing maternal healthcare continuum and suggest for optimizing utilization of skilled maternal healthcare services. The interviews were conducted in both the local language -Luo and English, using semi structured interview guide. During the interview follow up questions using probes were asked in order to acquire a deeper understanding when an explanation was unclear. The interviews lasted on average, 1 hour. All interviews were recorded, translated and transcribed verbatim in English. 
Table 1:- Themes and categories of data analysis

\begin{tabular}{|c|c|}
\hline hemes & categories \\
\hline 1.Knowledge on maternal health & $\begin{array}{l}\text { - Awareness of availability of skilled maternal healthcare } \\
\text { services } \\
\text { - Awareness of importance of skilled maternal healthcare } \\
\text { services }\end{array}$ \\
\hline $\begin{array}{l}\text { 2.Perception of barriers to utilization of } \\
\text { skilled maternal healthcare services }\end{array}$ & $\begin{array}{l}\text { Supply side barriers } \\
\text { - Availability of skilled maternal healthcare services } \\
\text { - Access to skilled maternal healthcare services } \\
\text { - Providers attitude } \\
\text { Demand side } \\
\text { - Availability of Community maternal healthcare services } \\
\text { - Access to community maternal healthcare services } \\
\text { - Status of maternal healthcare continuum } \\
\text { - Mothers fears } \\
\text { - Confirmation of pregnancy, labour and delivery time } \\
\text { - Transport and cost } \\
\text { - Availability of traditional birth attendants }\end{array}$ \\
\hline $\begin{array}{l}\text { 4. Perceived strategies to enhance } \\
\text { continuum of maternal healthcare } \\
\text { services }\end{array}$ & 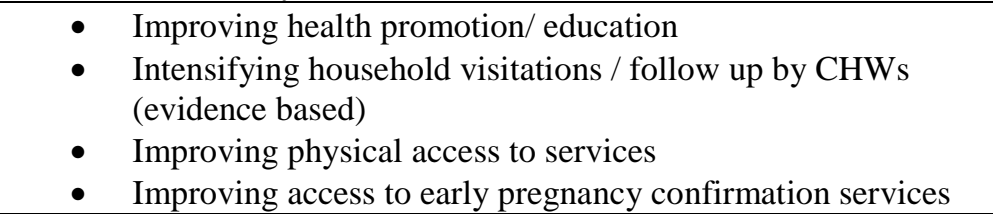 \\
\hline $\begin{array}{l}\text { 5. Perceived strategies to optimize } \\
\text { utilization of skilled maternal healthcare } \\
\text { services }\end{array}$ & $\begin{array}{l}\text { - Improving awareness on importance of skilled maternal } \\
\text { healthcare services } \\
\text { - } \quad \text { Improving availability of skilled maternal healthcare } \\
\text { - } \quad \text { Improvices } \\
\text { - Improving access to skilled maternal healthcare services } \\
\text { Improving health infrastructure and supplies } \\
\text { - Provision of incentives to women (Nets, Mother baby packs } \\
\text { etc) }\end{array}$ \\
\hline
\end{tabular}

The key informant in depth interviews were also conducted to 14 health providers who were working at the link health facilities. The providers were asked about the maternal healthcare services that they provide at the health facility, the available innovations to increase utilization of skilled delivery services, suggestions for enhancing care continuum and optimizing utilization of skilled delivery services. The interviews were conducted in English. During the interviews, follow up information using probes in order to acquire deeper understanding when an explanation was unclear. The interviews lasted on average, 30 minutes.

\section{Data analysis:-}

The facilitators took note of the respondent characteristics; influence by other participants; context within which the comments were made; internal consistency - for example changes in opinion of influence by other participants; frequency and extensiveness; specificity of comments, such as personal experience or hypothetical situation; intensity of comments, like depth of feeling; relationship with other criteria. Therefore in the analysis of individual opinions, the opinions that changed due to group dynamics, as well as opinion of groups expressed on the basis of consensus, were identified. All recorded discussions were transcribed verbatim in full. The data analysis focused on developing coding categories where narrative information was organized according to emerging themes using thematic analysis [24]. During content analysis [25, 26], the researcher used a deductive approach to identify users and providers perception on accessing care according to the three delay models of 1) seeking care, 2) reaching and 3 ) receiving care. A fourth theme was also added based on the supply demand interface to describe perceived strategies to increase utilization of skilled maternal healthcare services (Table 1). We then added the content of the FGD notes according to the themes of analysis. Finally all data was organized thematically and summarized according to pattern of findings. 


\section{Ethical considerations:-}

Before conducting each FGD, we explained the nature of the study, its rationale, and the extent of involvement expected from the participants. All respondents signed a written focus group consent form before participating in the FGD. A witness read the informed consent form to illiterate individuals, and those who consented to participate placed their thumbprint on the form, which was signed by the witness. The University of East Africa at Baraton Ethical Review Committee gave approval for this study (REC: UEAB/05/02/2015)

\section{Results:- \\ Knowledge on maternal health:- \\ Awareness of availability of skilled maternal healthcare services:-}

Women's awareness of the importance of skilled maternal health services during pregnancy, delivery and after child birth is important to enable them make informed decisions. Women need to understand that complications may occur without warning anytime during pregnancy, labour and even after having a normal delivery; hence it is safer to be in a setup where emergencies can be handled effectively. Information from the key informants revealed that antenatal care, skilled delivery services and postnatal care services were being offered in all the selected link health facilities. The findings showed that many women were aware that antenatal and skilled delivery services were available at their link health facility. However, very few knew that there were services being offered to women after deliver (Postnatal care).

Further probing as to whether they received any services after delivery revealed that apart from a few who could recall having been asked how they were fairing on after delivery, the majority knew that the services belonged to the baby. In six out of the eight focus groups, maternal healthcare services were reported to be irregular. In Ndori and Nyawita skilled maternal healthcare services were reported to be offered 24 hours a day, while in Ligala, West Katweng'a, and Yiro West, the participants reported that the skilled maternal healthcare services were being offered only during the day from Monday to Friday (8am to 5pm). The findings also revealed that in Marenyo and Gongo the services were not available during the weekends and nights. One 23 year old service user at Marenyo FGD said "In our health facility, delivery services are offered only during the day but at night, there is no staff. The worst is over the weekend when the facility is closed". Further clarity from the health providers confirmed these findings and attributed them to shortage of staff and lack of staff houses within the health facility. One service provider said "We are sometimes not able to give adequate information to the mothers because of staff shortage and too much work load"

Awareness of importance of skilled maternal healthcare services and risks in pregnancy:Interestingly, though the majority knew about the availability of antenatal and skilled delivery services, the majority were unable to articulate the importance of the skilled services. In most of the focus groups, the participants were able to mention health education, weighing, checking of blood pressure, abdominal examination, checking of blood level (signs of anaemia) and treating the sick women. It was interesting to note that when asked to spell put the importance of specific services offered, most respondents were quite and did not want to explain, an indication of not being sure. Even when asked to explain why weighing was being carried out, the majority shared that it was done to check whether the baby was growing well.

Another finding was that many women knew the samples that were being tested during pregnancy like blood and urine. However, the majority were not sure about the key investigations that were being carried out. One 39 years old service user from Ligala focus group said "To be frank, I am not sure of what is checked in the blood". However some indicated that it was done to check if they had enough blood, or for HIV test, or Malaria testing, with only two groups mentioning testing gonnorrhoea and sexually transmitted infections. In addition although participant mentioned that height was also being taken but they did not know the reasons why it was being taken. One 32 year old service user from Umalla Focus group said "I don't know why it is done" and the rest of the discussant were in agreement and were nodding.

In the opinion of key informants, awareness of skilled maternal healthcare services is usually emphasized during health education at the antenatal clinics and during household visitations by community health workers. The above findings showed that the women had inadequate knowledge on maternal health. The findings also revealed that there were hiccups in quality of user - provider interaction which was evident when women were unable to explain the exact care provided to them. 
With regard to the knowledge on risks in pregnancy, Women are expected to have knowledge of the risks to enable them make informed decisions. The discussants were therefore asked to mention the danger signs to observe during pregnancy and after child birth. The findings revealed that the majority of the discussants were able to mention only a few danger signs in pregnancy. The responses were varied in all the discussion groups as no group was able to mention all the key danger signs. Those mentioned included: bleeding, headache, palpitations, lack of foetal movement and dizziness. It was noted that danger signs such as epigastric pain and drainage of liquor were not known to the women even though they are also life threatening. Although the majority knew that bleeding during pregnancy was a danger sign, there were varied responses on the amount of bleeding that was considered to be dangerous. Further probing showed that some participants thought that only severe bleeding was dangerous. One 30 year old service user from Gongo focus group said "When you see bleeding that flows to the ground, know that you are in danger". These findings indicate that women understanding of danger signs were inadequate. The service providers revealed that this may be partly due to lack of proper education about danger signs by community health workers. One service provider from Bondo Hospital said "I think the skilled providers do assume that CHWs have educated the mothers when this is actually not the case. Even the CHWs themselves need the education". These data indicate that there is a need to retrain the community health workers and women of reproductive age on pregnancy danger signs.

\section{Perception of barriers to utilization of skilled maternal healthcare services:-}

The study revealed that though the government had made several strides to address barriers to utilization of skilled maternal healthcare services, there were still many supply and demand side barriers that were effecting utilization of these services. Some of the barriers included:

\section{Supply side barriers:- \\ Service availability and access:-}

Availability of services was translated to mean that services were being offered 24 hours a day. The women identified the consistent service availability as the enabling factor for utilization of skilled maternal healthcare services. The study found out that service availability and access was a key issue in most of the discussion groups particularly shortage of skilled providers, and lack of night and weekend services which was cutting across in six (6) out of the eight (8) health facilities. Some participants shared that they could go to the health facility at night only to find the place closed. One 20 year old from Umala focus group said "One day I went to the hospital at 2 pm on Saturday after hiring a motorcycle and I was feeling headache but only to reach the facility and I found the gate closed. I had to request the motorcycle operator to take me to Siaya district hospital and when I reached there; my blood pressure was found to be very high. I was very frustrated and it was too expensive for me". Similarly, health service providers from Umala, Sikalame, Ligala and Chianda mentioned lack of 24 hours maternal healthcare services, lack of staff houses/ call rooms and negative provider attitude were the major barriers to skilled maternal healthcare services.

\section{Attitude of Providers:-}

In all the FGDs carried out, negative service providers' attitude was cited as a hindrance to utilization of skilled maternal healthcare services. Service availability depends on health workers attitude. An example of the poor attitude is illustrated by the following quote by an 18 year old service user from Nyawita focus group who said "If you are unlucky to find those harsh ones, you will regret why you found yourself there" and probed further to state why they she would regret she said "Heh....Heh..., you can really be shouted at!". Contrary to the views of many discussants that the health staff had negative attitude, in Ligala focus group, a previous user of skilled health services was quick to tell her fellow discussants to stop generalizing that health staff have negative staff attitude because she had not had such experience despite having delivered her two children at the health facility.

\section{Demand side barriers:-}

\section{Awareness of availability of community maternal healthcare services:-}

Although the majority of participants reported being aware of some of the services being provided by community health volunteers (CHWs), further probing showed that they could not distinguish between services offered to all the general populace and those that are specific to maternal health. In seven (7) out of eight (8) focus groups, the discussants shared that the following services were being offered: health education on nutrition, hygiene and breastfeeding, testing for Malaria, treatment of malaria, testing for HIV and treatment of diarrhea were services being offered by community health volunteers. Some participants mentioned massaging of the abdomen and changing of the baby's position as also being done by traditional birth attendants (TBAs). Contrary to the views of 
other discussants, in Yiro focus group, four participants were surprised to hear that there were maternal healthcare services being offered at the community. They said that they had never received any maternal healthcare services from the community health volunteers. Some women from Ligala focus group commented that "May be some have been receiving these services, us we have never received any maternal healthcare services from CHWs"

\section{Access to community maternal healthcare services:-}

Most of the discussants had not received any maternal healthcare services from the community health volunteers while for the few who acknowledged having received such services, they generally mentioned health education on importance of utilizing skilled maternal healthcare services but were not specific to issues such as education on danger signs in pregnancy, individual birth plan, nutrition of the mother and exercises among others which fall within the mandate of the community health volunteers. Moreover our study found out that for those who had received some form of community maternal healthcare services, none had records of the services provided at the household. All indicated that the records were with the community health volunteers. Differences in opinion between the providers and the mothers were found regarding the perception of community maternal healthcare services. Whereas in five focus groups mothers shared that they had never received any such services, the providers mentioned that the community maternal healthcare services were available.

\section{Status of maternal healthcare continuum:-}

With regard to continuum of care as described in PNMCH, 2010 "Saving lives depends not only on high coverage but also on the quality of care delivered through the continuum". In order to establish the status of maternal healthcare continuum, discussants and interviewees were asked to share their experience in service continuity at the household level. Findings from the study showed that there was no evidence of networking between the skilled providers and the Community Health Workers (CHWs). The findings showed that there were no instances where a discussant was referred to the CHWs by skilled health providers for maternal healthcare continuum. Similarly, when key informants (health providers) were asked whether there are occasions where they referred women who need follow up to the respective $\mathrm{CHWs}$, the majority said that they had not referred any case.

In trying to find out whether there was mechanism of knowing which health services had been given at the household level, it was established that there was no tool or booklet for documentation of health services provided at the household level for purposes of continuum of healthcare. One 24 year old service user from Yiro west focus group commented that "What normally happens is that everyone who visits the household offer services they feel is appropriate without reference to what the other person did. In fact sometimes you even find two providers visiting you in a day with the same health information or services. Like one day a CHW came and gave me a net, after about 3 hours, another health provider working with CDC also came and gave me a net after sharing with me information on prevention of malaria in pregnancy. I had to take both because I had other people to give."

\section{Mother's fears:-}

Fear of embarrassment, fear of being despised by the providers or being left alone among others. In one FGD it was revealed that being left alone during labour is one reason why women prefer to deliver at the traditional birth attendants (TBAs) place. The focus group discussions revealed that sometimes women end up delivering at home if they do not have the basic requirements needed because they fear being embarrassed. One 36 year old user at Gongo focus group said "Imagine when you are wrapping your baby with rags and others are having new clothes? That's why me I fear hospital delivery since at the TBAs place, nobody looks at you". Another 34 year old user at Ndori focus group said "Sometimes you don't have cotton wool and you plan to use pieces of clothes, you can't carry them to the hospital, you fear embarrassment by the health providers and other women". Consistent with the above finding; a key informant (skilled health provider) from Akala health center confirmed that they do inform pregnant women to have individual birth plans which includes; having at least two body wrappers, cotton wool or pads, a pair of gloves, warm baby wrappers, a cap and pair of socks, finances for upkeep, transport and Identifying Birth Companion.

Some of the women preferred home deliveries for fear of being left alone in labour ward. One 23 year old user at Yiro West focus group said "My friend delivered alone in the health facility and the nurse came to assist after the baby was already out. The reason was that she was still attending to the outpatients". Although various negative experiences were shared in various focus groups, In Ligala focus group, one 33 yaer old women from Nyawita FGD said "I have never experienced any negative experience shared above; I think some are just hearsay and we should 
not generalize. Skilled maternal healthcare services are what will save most women from complications or even dying".

\section{Uncertainty about pregnancy:-}

Analysis of discussions from various focus groups revealed that the reason why women receive the recommended four (4) antenatal care visits is that they start antenatal visits when it is too late after being sure that they are pregnant. The discussants in most focus groups highlighted the need to have pregnancy tests kits availed to CHWs so that the services can be accessible for confirmation of pregnancy once periods are missed. One 19 year old service user at Marenyo focus group said "I suggest that the CHWs are even given those pregnancy test kits to help women confirm pregnancy at home". A key informant at Bondo hospital also echoed similar sentiments that availing pregnancy testing services at the community would help in confirmation of pregnancy early and early initiation of ANC visits.

\section{Uncertainty about labour and time of delivery:-}

Some discussants shared that sometimes it's about not knowing the expected time of delivery especially in the first pregnancy or when a one has not been attending the antenatal clinic. Some persevere and by the time they realize that they are in labour, the baby is out. An incident was shared in Ndori focus group that an adolescent who did not attend antenatal clinic and did not know that she was in labour ended up delivering at home alone and then bled to death. It is important that women should be encouraged to utilize skilled maternal healthcare services and CHWs trained on pregnancy danger signs. One 39 years old user in Ndori focus group said "I think the CHWs should be trained on the signs of pregnancy so that they can identify the young girls who are pregnant and refer for confirmation"

\section{Transport and cost:-}

In all the FGDs, it was mentioned that pregnant women rely on motorcycle as the main means of transport. However, a majority of the discussants indicated that at night the fares were comparatively high. One 28 year old user in Umala focus group said "In our community, of late motorcyclists fear moving out at night as they risk being robed of their motorcycles and since most of the deliveries occur at night, women end up delivering at home". While another 30 year old service user from Marenyo focus group said "The use of motorcycles at night is also expensive because they overcharge and it is therefore not affordable". These data indicate that transport is a major barrier to utilization of maternal health services especially in rural communities.

\section{Availability of Traditional birth attendant's:-}

Another barrier to utilization of maternal health services was the presence of TBAs also in the communities. This was found to be contributing to increased number of home deliveries in the study area. One 29 year old from Marenyo focus group commented that "The fact that women have an alternative person within reach whom they know can conduct deliveries also make them to be opt for home deliveries" The above findings show that various barriers still exist and they affect utilization of skilled maternal healthcare services

\section{Perceived strategies for enhancing maternal healthcare continuum:-}

For purposes of designing strategies which are evidence based, effective and acceptable to both the women and the providers; the participants were asked to give their suggestions on the interventions that would enhance care continuum and increase utilization of skilled maternal healthcare services. The findings show that the majority of the participants felt that the key interventions that could enhance maternal healthcare continuum and optimize utilization of skilled maternal healthcare services were health facilities to offer 24 hours skilled delivery services and providers to have positive attitude. Others in order of their strength included: 1) CHWs to intensify awareness creation on maternal healthcare and importance 2) Helping women to confirm pregnancy early to avoid delays due to uncertainty, 3) Provision of incentives to women who complete $4^{\text {th }}$ antenatal care visits (Nets, Mother baby packs etc), 4) Developing ways of reminding women when clinic days are due and 5) Women to have birth companions (CHWs, TBAs or relatives).

Perceived strategies for optimizing utilization of skilled maternal healthcare services:-

Service providers suggested that in order to increase utilization of skilled maternal healthcare services. The followings need to be in place: Consistent availability of maternal healthcare services for 24 hours. The other suggestions which were cited in six focus groups and above included; Strengthen community-facility linkage and demand creation; Develop mechanisms for monitoring $\mathrm{CHVs}$ performance to ensure households information on 
maternal health; Provision of incentives to mothers who perform well and early identification of pregnant mothers by carrying out household pregnancy testing for suspected cases. Others included building of staff houses to accommodate health providers within the health facility and staff to stay at the facility, recruitment of additional health providers to provide skilled maternal health services, Positive attitude from healthcare providers, Reorient TBAs on new roles and Renovation/ Construction of maternity units at the facility.

\section{Discussion:-}

Increased utilization of skilled maternal healthcare services greatly reduced maternal morbidity and mortality [2, 27, 28]. This can be greatly improved through increased knowledge on maternal health services provided by skilled care providers [29]. However, one of the major barriers to utilization of skilled maternal healthcare service is lack of awareness of the importance of skilled maternal health services [30-33]. More importantly data reveals that although antenatal care, skilled delivery services and postnatal care services were being offered in all the link health facilities and many women were aware about these services, puzzling was that very few women knew about services being offered to women after delivery (Postnatal care). These data indicate there is a need to educate women about the importance of both antenatal and postnatal services provided in health facilities to increase the uptake of these services.

Lack of awareness about the importance of skilled maternal health services is another important barrier to utilization of these services [34]. A study in Tanzania revealed that the low utilization of skilled maternal health services was partly due to women not aware of the services available and the risk involved in traditional birth practices [35]. In Indonesia women use skilled maternal health services mainly due to childbirth complications [36]. This study shows although women knew that health education, weighing, checking of blood pressure, abdominal examination, checking of blood level and treating the sick women were some of the services provided as a component of skilled maternal health services, a majority thought that post-natal care services were for the baby. More importantly although a majority knew about the availability of antenatal and skilled delivery services they were not able to articulate the importance of the skilled services. These data indicate that women still do not understand the importance skilled maternal health services provided during antenatal and postnatal period despite importance of skilled maternal healthcare services being emphasized during health education at the antenatal clinics or during household visitations by community health workers. Overall these data indicate that there are challenges in the quality of user-provider interaction and there is a need to develop a more focused and interactive strategies to improve women literacy about the importance of skilled maternal health services. Indeed utilization of skilled maternal health services can be improved through a focused and sustained health education [37]. In Kenya, it was revealed that women who were exposed to media were more likely to uptake this services [38], suggesting that apart from skilled health providers and CHWs there is need for health education and promotion on skilled maternal health services through the media. In addition it has been shown that providing both males and their pregnant women with health education improve health behavior than educating women alone [39]. Women should therefore be empowered with detailed information of all available maternal healthcare services for them to make informed decisions [34]. It is also critical that information on benefits of skilled maternal healthcare service and the risks of not using these services should be propagated using all the available communication channels (during first interaction at the maternal healthcare clinic, during household visitations by CHWs and through community dialogue meetings). As recommended in [36]; the home visitations for postnatal care services will greatly benefit women and it should be emphasized to women that postnatal period is a crucial period where mother need specialized care to prevent unnecessary deaths $[5,40]$.

Inadequate availability of skilled birth attendants was found to be a major barrier to utilization of skilled maternal healthcare services in Siaya County [34, 41]. Although the findings showed that the availability of maternal healthcare services varied from facility to facility with six out of the eight selected health facilities offering limited maternal healthcare (either no night services or weekend services). The key informants attributed the lack of $24 \mathrm{hrs}$ services to the shortage of staff houses within the healthcare facilities and shortage of skilled staff. Therefore, there is a need to invest in recruitment of more staff and building of staff houses within the health facility compound. Consistent with previous findings, negative provider attitude was identified as one of the barriers affecting utilization of skilled maternal healthcare services in the study area [16, 17]. Therefore, to increase utilization of skilled maternal healthcare services, health workers must be sensitized to be sensitive to women's situation and needs, so as to provide client friendly services to all women regardless of their status. Additionally, health workers must strive to provide quality skilled delivery services to women when who choose to deliver at the health facility as self deliveries deter women from using health facility in subsequent pregnancies [13, 42, 43]. As recommended by 
[13], comprehensive health promotion through awareness-raising, appropriate education of healthcare workers and regular support supervision should be scheduled to improve provider communication and address burnout during service delivery to resolve critical issues that are likely to trigger negative responses from health providers [13]. Where necessary, women should be encouraged to come with supportive birth companions to address women's concern of being left alone during labour in some health facilities [44]. Other major barriers included fear of embarrassment of not having presentable clothing for the baby and self, uncertainty about pregnancy and uncertainty about labour, and high cost of transport at night. As suggested by users and providers in this study; these later issues could be addresses by increasing access to community maternal healthcare services, early identification of pregnancy mothers by use of pregnancy testing at the community and referring for initiation of $1^{\text {st }}$ ANC and educating the mothers on the early signs of labour. Further exploration should be made on developing an application for motorcycle drivers to transport mothers for delivery with possible modification of their seats to make them more comfortable.

Continuum of care is needed throughout pregnancy, childbirth and the postnatal period in order to improve maternal and newborn health and reduce morbidity and mortality $[45,46]$. Our data reveal that there is a weak health facilitycommunity linkage with no evidence of maternal healthcare continuum which could be attributing to low utilization of skilled maternal healthcare services. The findings also showed that there were no evidence or records at the household level to show that there were maternal healthcare services provided at the household by community health volunteers. Therefore, to enhance maternal healthcare continuum, there is a need to develop the following: (1) A tool (household health visitors log book) for recording all healthcare services provided at the houses hold and for reference purposes (2) Strengthen the use of referral tools for forward and backwards referrals to enhance informational and management continuity (3) Provide files for individual CHWs at the link health facility where records of all CHWs activities and referral will be kept for reference and performance tracking. Of importance is also to develop and distribute maternal health education protocols/ counseling cards with key messages for CHWs reference during household visitations and intensify supervision [47-49].

As suggested by previous studies instead of investing in supply-increasing interventions only, policies aiming at increasing access and utilization should take on both a supply- and demand side approach [50-52]. As Kerber et al. 2007 put it; strengthened linkage between the community and health facility is important in increasing utilization of skilled health services [53]. Of interest is that most suggestions proposed by the maternal healthcare service users and providers as measures to increase utilization of skilled maternal healthcare services could be used to address most of the barriers discussed in the focus groups. The key strategies proposed included: Developing mechanism for follow up of women from conception until post delivery, Provision of incentives to women who complete $4^{\text {th }}$ antenatal care visits (Nets, Mother baby packs), CHWs to be provided with tools/ maternal healthcare reference materials for health education, CHWs to be empowered to carryout pregnancy tests at the household for early identification of pregnant women, positive provider attitude and strengthening community-facility linkage. These suggestions could be used to address context specific causes of variable use of maternal healthcare if safe motherhood is to become a reality.

\section{Strengths and Limitations:-}

This study provides data on user's perception of skilled maternal healthcare services and suggestions for optimizing utilization which could inform policy makers to develop frameworks for optimizing utilization of skilled maternal health services. The sample selection methodology, the sample size and the inclusion of all samples from all the six sub counties in the County allows generalization of the results of this study [54]. The use of different of respondents increases the validity of the study [55].

Having one focus group in each sub county was limited by budget constraints and that could have influenced the number of established criteria. This limitation was offset by conducting a literature research with the aim of including all known important aspects. The number of participants in each focus group discussion was 9 to 12 participants this could have limited the confidentiality and anonymity of study participants because they all listened and interacted together. A large group would have limited the detail of some responses because participants feel a pressure to share airtime with others. Conversely, participants in a smaller group could feel an uncomfortable pressure to talk more than they would otherwise to cover up [56, 57]. However, this limitation was explained to the study participants before informed consent was obtained. Moreover, we reserved rooms in the link facilities to maintaining FGD confidentiality and enable study participants to talk freely and privately without others listening in. 


\section{Conclusion:-}

This study found out that users perception influence utilization of skilled maternal healthcare services. Consistent with previous studies, inadequate awareness of availability of maternal healthcare services and importance, limited availability of skilled maternal healthcare services, negative provider attitude, and weak facility-community linkage negatively influence utilization of maternal healthcare services. Our results suggest the need to have programs understand the perspectives of service users and providers for evidence based context specific interventions. Concerted efforts should be geared towards intensifying awareness creation on available of skilled maternal healthcare services and importance, availing 24 hours skilled delivery services and promotion of positive attitude amongst skilled providers. Critical is also the empowerment of community health volunteers to identify of pregnant mothers early and refer, provide basic maternal education and healthcare services at the community and instituting mechanisms for regular mentorship of CHWs and performance tracking. This study also highlights the views and suggestions from insiders (users and providers) which are explicable and should be considered in designing context specific evidence based interventions.

\section{What is already known on this topic}

Many developed countries including Kenya are still struggling to meet the set millennium development goal 5 on improving maternal health by increasing utilization of skilled maternal health care services especially $4^{\text {th }}$ antenatal care, skilled delivery and postnatal care services within 48 hours of birth. Experience over the past decade has shown that no single intervention is by itself sufficient to improve maternal and newborn health and reduce morbidity and mortality. What is needed is a continuum of care throughout pregnancy, childbirth and the postnatal period $[58,59]$. Context specific, evidence based interventions are therefore needed to increase utilization of skilled maternal healthcare services.

\section{What this study adds}

The findings reveal that most women in Siaya County do not know about the availability and importance of postnatal care services, and that the majority also do not have access to community maternal healthcare services. Concerted efforts should therefore be geared towards intensifying awareness creation on postnatal care services and importance, availing 24 hours skilled delivery services, provision of incentives to mothers and promotion of positive attitude amongst skilled providers in order to increase uptake of skilled maternal healthcare services. Important is also empowerment of community health volunteers (CHVs) ) to be able to identify pregnant mothers early through pregnancy testing at community and enhancing maternal healthcare continuum by providing evidence based community maternal health services.

\section{List of abbreviations:-}

WHO: World health organization; FGD: Focus Group Discussion; CHWs: Community Health Workers; TBAs: Traditional Birth Attendants CU: Community Units; ANC: Antenatal Clinic; MDG: Millenium Development Goals; PNMCH: Partnership for Newborn, Maternal and Child Health; KNBS: Kenya National Bureau of Statistics; UEAB: University of Eastern Africa Baraton; ANC: Ante Natal Care; MOH: Ministry Of Health; RMNCH: Reproductive Newborn, Maternal and Child Health

\section{Declarations:-}

\section{Acknowledgements:-}

Authors would like to acknowledge Department of Health, Siaya County, Skilled health providers and community Health workers in Siaya County, Research assistants, and all the participants who participated in this study.

\section{Ethical considerations:-}

Ethical clearance was obtained from Research Ethics Committee of University of Eastern Africa, Baraton, Eldoret (REC: UEAB /05/02/2015). The research assistants were trained on research ethics. Informed written consent from the study participants was obtained and the objective of the study was explained to them. Privacy and confidentiality of collected information was ensured at all levels.

\section{Consent to publish:-}

Consent to publish was obtained from Research Ethics Committee of University of Eastern Africa, Baraton, Eldoret (REC: UEAB /05/02/2015). 


\section{Competing interests:-}

The authors declare that there are no competing interests.

\section{Funding:-}

This work was supported by kind contributions from friends.

\section{Author Contributions:-}

OEO conceptualized the paper, searched literature, and wrote the manuscript draft. ASO contributed to the design of the study and provided advice regarding data interpretation. FAA helped develop the data analysis framework and also helped train field researchers for data collection. OEO and ASO analyzed the qualitative data. AK made critical revisions to the paper and provided advice regarding data interpretation. OSO participated in data collection and helped write the results and discussion sections. All authors read and approved the final manuscript.

\section{Author details:-}

Department of Public Health, School of Health Sciences, Jaramogi Oginga Odinga University of Science and Technology ${ }^{1}$; Department of Biological Sciences, School of Biological and Physical Sciences, Jaramogi Oginga Odinga University of Science and Technology ${ }^{2}$; Department of Health, County Government of Siaya ${ }^{3}$

\section{References:-}

1. Abou-Zahr CL, W.T., Antenatal care in developing countries:promise, achievements and missed opportunity: an analysis of trends, levels and differentials, 1990-2001. World Health Organization,Geneva, 2003.

2. Erin Sines, U.S., Steve Wall, and Heidi Worley, Postnatal Care: A Critical Opportunity to Save Mothers and Newborns. 2007.

3. WHO, U., UNFPA, The World Bank and the United Nations Population Division., Trends in Maternal Mortality: 1990 - 2013. 2014.

4. Conde-Agudelo A, B.J., Lammers C, Maternal-perinatal morbidity and mortality associated with adolescent pregnancy in Latin America: Cross-sectional study. American Journal of Obstetrics and Gynecology, 2004. 192: p. 342-349

5. Wang W, A.S., Wang S, Fort A, Levels and Trends in the Use of Maternal Health Services in Developing Countries, in DHS Comparative Reports. 2011: Calverton, Maryland.

6. Carroli G, R.C., Villar J, How effective is antenatal care in preventing maternal mortality and serious morbidity? An overview of the evidence. Paediatric Perinatal Epidemiology, 2001. 15(s1): p. 1-42.

7. WHO, I.a.F., Making pregnancy safer: The critical role of the skilled attendant 2004.

8. ADAMSON, P.e.a., Marginalized women are being left behind ?: a population-based study of institutional deliveries in Karnataka, India. BMC Public Health, London, 2012. 12(30).

9. Agha S, C.T., Determinants of institutional delivery in rural Jhang, Pakistan. International journal for Equity in Health, 2011. 10(1): p. 31.

10. Baral YR, L.K., Skinner J, van Teijlingen ER, Determinants of skilled birth attendants for delivery in Nepal. Kathmandu University Medical Journal 2010. 8 (No.3)(31): p. 325-32

11. Statistics, K.N.B.o., Nyanza Province Multiple Indicator Cluster Survey 2011, Final Report. 2013, Kenya National Bureau of Statistics,Nairobi, Kenya:.

12. Macro, K.N.B.o.S.K.a.I., Kenya Demographic and Health Survey 2008-09, in DHS Final Reports. 2010: Nairobi, Kenya.

13. Simkhada BD, V.T.E., Porter M, Simkhada P, Factors affecting the utilization of antenatal care in developing countries: systematic review of the literature. Journal of Advanced Nursing, 2008. 61(3): p. 244-60.

14. Chakraborty N, I.M., Chowdhury RI, Bari W W, Akhter HH, Determinants of the use of maternal health services in rural Bangladesh. . Health Promotion International, 2003. 18(4): p. 327-337.

15. Health, M.o., District Health Information Software (DHIS 2). 2014.

16. Global Health Workforce Alliance, W.H.O., Global Experience of Community Health Workers for Delivery of Health Related Millennium Development Goals: A Systematic Review, Country Case Studies, and Recommendations for Integration into National Health Systems. 2010: WHO.

17. Pell C, M.A., Were F, Afrah NA, Chatio S, et al., Factors Affecting Antenatal Care Attendance. PLoS ONE 2013. 8(1).

18. UNICEF, S.C.H.M.T., Siaya Investment Case for Maternal and Child Health. 2013, County Government: Siaya County. 
19. KNBS, Kenya National Bureau of Statistics (KNBS), ICF Macro. 2010. Kenya. Demographic and Health Survey 2008-2009. Calverton, Maryland: KNBS, ICF Macro. 2009.

20. Kenya National Bureau of Statistics - KNBS, Kenya Demographic and Health Survey 2008-09. 2010, KNBS and ICF Macro: Calverton, Maryland, USA.

21. Bottleneck, Bottleneck analysis 2012; Siaya investment case for MNCH. 2013.

22. Pell C, M.a.A., Were F, Afrah NA, Chatio S, Factors Affecting Antenatal Care Attendance: Results from Qualitative Studies in Ghana, Kenya and Malawi. PLoS ONE, doi:10.1371/journal.pone.0053747, 2013. 8(1): p. e53747.

23. Siaya Health Department, U., Siaya Investment Case for Maternal, Newborn and Child Health; Bottleneck analysis, 2013.

24. Braun, V.a.C., V., Using thematic analysis in psychology. Qualitative Research in Psychology,. 3, 2006. 2: p. 77-101.

25. Berkowitz, S., Analyzing Qualitative Data. In J. Frechtling, L. Sharp, and Westat (Eds.), UserFriendly. Handbook for Mixed Method Evaluations(Chapter 4). 1997.

26. Bogdan R. B. \& Biklin, S.K., Qualitative Research for Education: An Introduction to Theory and Methods, Third Edition. Needham Heights, MA: Allyn and Bacon. 1998.

27. AbouZahr C, W.T., Antenatal Care in Developing Countries: Promises, Achievements and Missed Opportunities - An Analysis of Trends, Levels and Differentials, 1990-2001.Geneva. 2003, World Health Organization: 20 Avenue Appia, 1211 Geneva, 27, Switzerland.

28. Darmstadt GL, B.Z., Cousens S, Adam T, Walker N, de Bernis L, Lancet and N.S.S. Team, Evidence-based, cost-effective interventions: how many newborn babies can we save? Lancet, 2005. 365(9463): p. 977-988

29. MAHITI, G.R.e.a., Women's perceptions of antenatal, delivery, and postpartum services in rural Tanzania. . Global Health Action, 2015. 8(ISSN 1654-9880. ).

30. Sandberg J, O.P.K., Asp G, Kabakyenga J, Agardh A Inadequate Knowledge of Neonatal Danger Signs among Recently Delivered Women in Southwestern Rural Uganda: A Community Survey. PLoS ONE 2014. 9(5).

31. Mpembeni RN, K.J., Leshabari MT, Massawe SN, Jahn A, Mushi D, et al. , Use pattern of maternal health services and determinants of skilled care during delivery in Southern Tanzania: implications for achievement of MDG-5 targets. BMC Pregnancy Childbirth, 2007. 7(29).

32. Tsawe, M., \& Susuman, A. S. , Determinants of access to and use of maternal health care services in the Eastern Cape, South Africa: a quantitative and qualitative investigation. BMC Research Notes, 2014. 7(723).

33. Shahram, M.S., Hamajima, N., \& Reyer, J. A., Factors affecting maternal healthcare utilization in Afghanistan: secondary analysis of Afghanistan Health Survey 2012. . Nagoya Journal of Medical Science, 2015. 77(4): p. 595-607.

34. Sharad Onta, B.C., Binjwala Shrestha, Narayan Subedi, Gajananda P. Bhandari, andAlexandra Krettek, Perceptions of users and providers on barriers to utilizing skilled birth care in mid-and far-western Nepal: a qualitative study. Globa Health Action, 2014. 7(10).

35. Mpembeni RN, K.J., Leshabari MT, Massawe SN, Jahn A, Mushi D, et al. , Use pattern of maternal health services and determinants of skilled care during delivery in Southern Tanzania: implications for achievement of MDG-5 targets. BMC Pregnancy Childbirth, 2007: p. 7:29. .

36. Titaley CR, H.C., Dibley MJ, Heywood P. , Why do some women still prefer traditional birth attendants and home delivery? A qualitative study on delivery care services in West Java Province, Indonesia. BMC Pregnancy Childbirth., 2010: p. 10:43. .

37. Ononokpono, D.N., \& Odimegwu, C. O. , Determinants of Maternal Health Care Utilization in Nigeria: a multilevel approach. . The Pan African Medical Journal, , 2014. 17(Suppl 1),(2).

38. Van Eijk, A.M., Bles, H. M., Odhiambo, F., Ayisi, J. G., Blokland, I. E., Rosen, D. H., ... Lindblade, K. A., Use of antenatal services and delivery care among women in rural western Kenya: a community based survey. . Reproductive Health, , 2006. 3(2).

39. Britta C. Mullany, S.B.a.M.J.H., The impact of including husbands in antenatal health education services on maternal health practices in urban Nepal: results from a randomized controlled trial

40. Titaley CR, H.C., Dibley MJ, Heywood P, Why do some women still prefer traditional birth attendants and home delivery? A qualitative study on delivery care services in West Java Province, Indonesia BMC Pregnancy Childbirth, 2010. 10(43).

41. Esena, R.K.M.-M.S., Factors Associated with the Utilization of Skilled Delivery Services in the Ga East Municipality of Ghana Part 2: Barriers to Skilled Delivery. International Journal of Scientific and Technology Research, 2013. 2(8): p. 195-207. 
42. Gao J, Q.J., Tang S, Eriksson BO \& Blas E, Health equity in transition from planned to market economy in China. Health Policy Planning, 2002. 17(supplementary): p. 20-29.

43. Wild K, B.L., Kelly P, Martins N, Birth choices in Timor-Leste: a framework for understanding the use of maternal health services in low resource settings. Soc Sci Med, 2010. 71(11): p. 2038-45.

44. Banda, G.N., Female support companionship during labour: Effects on infant Apgar score and maternal pain experience, in University of Malawi,College of Medicine, Blantyre. 2008.

45. Gilbert Wangalwa, B.C., David Wamalwa, Yvonne Machira, Peter Ofware, Meshack Ndirangu, Festus Ilako, Effectiveness of Kenya's Community Health Strategy in delivering community-based maternal and newborn health care in Busia County, Kenya: non-randomized pre-test post test study. Pan Afr Med Journal, 2013. 13(supp 1)(12).

46. The Partnership for Maternal, N.a.C.H., From Hope to Action. 2010.

47. Nsabagasani X, J.N.S., Kallander K, Peterson S, Pariyo G, Tomson G. , Home-based management of fever in rural Uganda: community perceptions and provider opinions. . Malaria Journal, 2007. 6(11).

48. Scott H, D.I., Accountability for improving maternal and newborn health.. Elsevier Ltd., 2016.

49. Suri A1, G.K., Carpenter S, Voices from the field: perspectives from community health workers on health care delivery in rural KwaZulu-Natal, South Africa.Journal of infectious diseases, 2007. 1(196 (suppl.3)).

50. Hjortsberg, C., "Why do the sick not utilize health care? The case of Zambia"Health Economics, 2003. 12: p. 755-770.

51. Thaddeus, S., \& Maine, D, Too far to walk: maternal mortality in context. Social Sciences and Medicine, 1994. 38(8): p. 1090-1110.

52. Ahmed, S.A., A.; Chowdhury, M.; Bhuiya, A, "Gender, socioeconomic development and health-seeking behaviour in Bangladesh". Social Science and Medicine, 2000. 51: p. 361-371.

53. Kate J Kerber, J.E.d.G.-J., Zulfi qar A Bhutta, Pius Okong, Ann Starrs, Joy E Lawn, Continuum of care for maternal, newborn, and child health: from slogan to service delivery Lancet 2007. 370: p. 1358-69.

54. Olive m.Mugenda, A.G.M., Research Methods: Quantitative and Qualitative Approaches a. 2008 .

55. Tuckett, A., Qualitative research sampling-the very real complexities. Nurse Researcher, 2004. 12(1): p. 47-61.

56. 557-562., J.C.F.P., Doing qualitative research in General Practice: Methodological utility and engagement. Family Practice, 2002. 19(5): p. 557-562.

57. Wong, L.P., Focus group discussion: a tool for health and medical research. Singapore medical journal,, 2008. 49(3): p. 256-60.

58. Lassi, Z.S., Kumar, R., Mansoor, T., Salam, R. A., Das, J. K., \& Bhutta, Z. A. , Essential interventions: implementation strategies and proposed packages of care. Reproductive Health.. 2014. 11(Suppl 1)(S5).

59. Portela, A. and C. Santarelli, Empowerment of women, men, families and communities: true partners for improving maternal and newborn health. British Medical Bulletin, 2003. 67(1): p. 59-72. 